\title{
Regulation of Tension on Hair-Cell Transduction Channels: Displacement and Calcium Dependence
}

\author{
Nir Hacohen, ${ }^{1}$ John A. Assad, ${ }^{1,2}$ Wendy J. Smith, ${ }^{1, a}$ and David P. Corey ${ }^{1,3}$ \\ 'Neuroscience Group, Howard Hughes Medical Institute, and Department of Neurology, Massachusetts General Hospital, \\ Boston, Massachusetts 02114, and 'Department of Neurobiology and ${ }^{3}$ Program in Neuroscience, Harvard Medical School, \\ Boston, Massachusetts 02138
}

\begin{abstract}
An epithelial preparation of the bullfrog sacculus was used to characterize the initial rate of the adaptation mechanism in hair cells and its dependence on displacement and calcium. The $K X$ ) curve relating transduction current and bundle displacement shifted along the $X$-axis without substantial change in slope, as previously observed, suggesting that adaptation involves a change in the attachment point of the elastic element connected to ion channels. If the "tip links" model of transduction is correct, this implies that one end of the link moves along the side of the stereocilium. The rates were highly asymmetric: in the tensioning direction the rate was roughly constant at $1-2 \mu \mathrm{m} / \mathrm{sec}$ (calculated as motion along a stereocilium); this is similar to that of myosin on actin. In the relaxing direction it appeared linearly dependent on tension. Calcium preferentially potentiated the relaxation, and apparently reduced the resting tension in the elastic element. The calcium site appears specific for calcium, as other divalent cations inhibited its action. Dihydrostreptomycin inhibited the positive rate, but its effect could not be explained by a simple channel block, and it seems inconsistent with screening of negative charge in the mouth of the transduction channel.
\end{abstract}

Tension exerted through some elastic linkage appears to be the essential stimulus for gating of transduction channels in vertebrate hair cells (Corey and Hudspeth, 1983b; Howard and Hudspeth, 1987). An attractive model supposes that fine filaments extending vertically from the tip of each stereocilium to the side of its taller, adjacent neighbor (the "strings" or "tip links") constitute the linkage to channels in the tips of stereocilia, and that lateral displacements of the stereocilia cause a relative shear at their tips to alter tension in the tip links (Hudspeth, 1982; Pickles et al., 1984). Because the transduction current is not zero at rest but is $20-40 \%$ of the maximum (Hudspeth and Corey, 1977), it is thought that some mechanism exerts a resting tension on transduction channels.

\footnotetext{
Received Dec. 30, 1989; revised June 2, 1989; accepted June 8, 1989.

We thank John L. Hines for machining optical components, Susan L. Cronin for laboratory administration, and Gordon M. G. Shepherd for useful comments on the manuscript.

This work was supported by National Institutes of Health grant NS-22059 (to D.P.C.), by Office of Naval Research grant N00014-88-K-0078 (to D.P.C.), and by the Howard Hughes Medical Institute.

Correspondence should be addressed to David P. Corey, Department of Neurology, Wellman 414, Massachusetts General Hospital, Boston, MA 02114.

a Present address: College of Physicians and Surgeons, Columbia University, New York, NY 10027.

Copyright (C) 1989 Society for Neuroscience $0270-6474 / 89 / 113988-10 \$ 02.00 / 0$
}

Following displacements that increase the current through transduction channels, the current declines towards the resting level over about $100 \mathrm{msec}$ (Corey and Hudspeth, 1983a). The decline can be described operationally as a simple shift, along the displacement axis, of the curve relating displacement and transduction current [the $I(X)$ curve]; in this model the shift would correspond to a relaxation of tension in the linkage (Eatock et al., 1987). Similarly, a displacement that reduces transduction current is followed by an increase in current towards the resting level, corresponding to a restoration of linkage tension. Howard and Hudspeth (1987) observed changes in the stiffness of stereociliary bundles with a time course that matched that of the change in transduction current, further supporting the model. There appears to be a mechanism, then, that acts to keep constant the tension in the linkages attached to the ion channels. What is the molecular mechanism of the tension regulation system?

The mechanism is known to be sensitive to calcium concentration in the medium around the stereocilia (Eatock et al., 1987). Because the calcium sensitivity disappears at positive membrane potentials, the calcium site-and presumably the mechanism itself-is thought to be intracellular (Assad et al., 1989). Because the voltage dependence occurs almost instantly, the site is thought to be close to the transduction channels (within 1-2 $\mu \mathrm{m}$ ) (Assad et al., 1989). Lastly, depolarization that increase the tension on ion channels, inferred physiologically, also cause motions of the hair bundles (Assad et al., 1989), suggesting that the tension-regulating mechanism is an active process that involves an energy-consuming motile element.

In this paper we report the displacement and calcium dependence of the mechanism. By measuring the initial rate of the mechanism we can infer some of its biophysical properties. These properties suggest that the mechanism is primarily sensitive to tension but is modulated by intracellular calcium. The results are consistent with a model (Howard and Hudspeth, 1987) that involves a change of the attachment point of the tip link, by a myosin-like molecule moving on the actin core of the stereocilium. A preliminary account of some of this work has appeared (Corey et al., 1987).

\section{Materials and Methods}

Microphonic preparation. We used the in vitro microphonic preparation of the bullfrog sacculus, essentially as described by Corey and Hudspeth (1983a, b) and by Eatock et al. (1987). Briefly, sacculi were dissected from bullfrogs (Rana catesbeiana) into a saline solution; the maculae were trimmed of surrounding tissue and mounted across a hole separating an apical and a basal chamber. Most of the otolithic membrane was peeled off hair bundles, leaving a patch of several hundred hair cells of similar orientation still attached in the periphery of the macula. Apical 


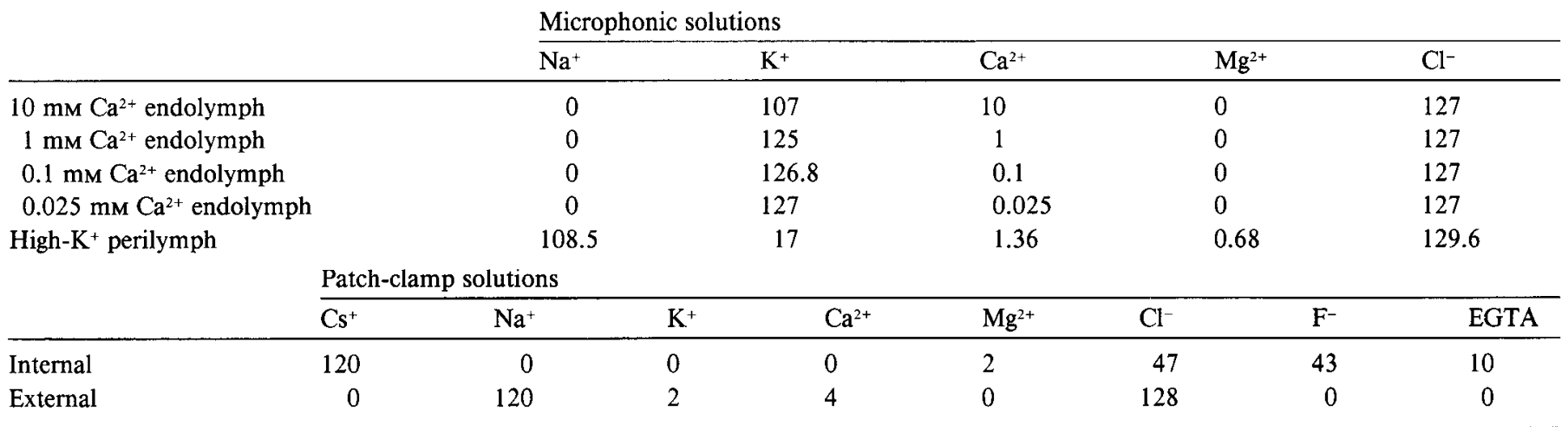

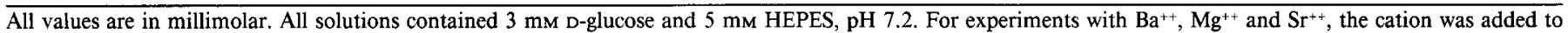
the $0.1 \mathrm{~mm} \mathrm{Ca}^{2+}$ endolymph as the chloride salt to a final concentration of $1 \mathrm{~mm}$.

and basal chambers were perfused continuously during an experiment. Under these conditions, the preparation was very stable for the first 3$4 \mathrm{hr}$ of recording, during which these data were recorded, and still responded at moderate amplitude $24 \mathrm{hr}$ later.

Stimulus. The otolithic membrane was moved by a glass probe of about $100 \mu \mathrm{m}$ tip diameter, driven in the plane of the macula by a piezoelectric bimorph element. The amplitude of the probe motion was calibrated against an eyepiece micrometer by observation at $1000 \times$; its time course was observed by focusing a laser at the probe tip and recording the light not scattered. The probe moved with a sigmoidal rise and fall of about $100 \mu \mathrm{sec}$ and ringing of less than $4 \%$ of the step amplitude. We have shown that the hair bundles follow the motion of the probe within about $25 \mu \mathrm{sec}$ (Corey and Hudspeth, 1983b).

Recording. The transepithelial current was measured with a voltage clamp that controlled the potential between the apical and basal chambers. The transepithelial voltage was set to a value that caused no transepithelial current in the absence of mechanical stimulation, usually within $5 \mathrm{mV}$ of zero. Series resistance compensation was not used for these experiments; consequently, the current signal was low-pass-filtered with a time constant equal to the product of the series resistance and the epithelial capacitance, about $60 \mu \mathrm{sec}$ (Corey and Hudspeth, 1983a). The output of the clamp was additionally filtered with a 4-pole Bessel filter, usually set at $2 \mathrm{kHz}$. The current signal was digitized at a rate of 50 $\mu$ sec per point and transferred to disk on a PDP $11 / 73$ computer (INDEC Systems).

Current flowing from apical to basal chamber, which corresponds to inward receptor current, is shown as upward in the figures. Although opposite to the normal electrophysiological convention, it simplifies comparison to earlier work with this preparation (Corey and Hudspeth, 1983a, b; Eatock et al., 1987) and matches the convention of showing increased channel open probability as upwards.

Solutions. Apical and basal chambers, bathing the hair cells' apical and basolateral surfaces, respectively, were separately perfused at a rate of $0.2-0.5 \mathrm{ml} / \mathrm{min}$. Apical solutions had a high potassium concentration, like endolymph, and a variable divalent cation concentration (see Table 1). The basal solution was always a perilymph-like solution with potassium elevated to $17 \mathrm{~mm}$ to depolarize cells to about $-50 \mathrm{mV}$, which is a low-resistance region of their $I(V)$ relation. Under these conditions, cells had very small receptor potentials, so that voltage-dependent currents did not substantially contribute to the mechanically activated current (Corey and Hudspeth, 1983a). In addition, the measurements were relatively immune to the contribution of voltage-dependent currents, because the assay for adaptation was the shift of the $I(X)$ curve rather than the change in the transduction current itself.

Temperature was monitored with a thermocouple probe in the apical chamber. All experiments were done at room temperature, between 21 and $22.5^{\circ} \mathrm{C}$.

Protocol and analysis. The experimental protocol was generally that of Eatock et al. (1987). The relationship between displacement stimulus and receptor current [the $I(X)$ curve] was measured with a series of short displacements (test steps) before a maintained displacement stimulus (the adapting step), and then measured again at various times during the adapting step (Fig. $1, A, B)$. We moved the initial $I(X)$ curve along the displacement axis to fit each shifted curve (Fig. 1C). For large shifts, the $I(X)$ curves broadened slightly, perhaps because different hair cells in the epithelium shifted by different amounts. In those cases, the shift was measured from the stcepest part of the $I(X)$ curves. This shift corresponds to the decline in the effective displacement reaching the transduction element.

The shifts at each time were then plotted against time for comparison to the adapting step (Fig. $1 D$ ), and an exponential curve fitted by eye to the points. The initial rate of the shift was measured as the initial slope of the exponential curve and plotted against the amplitude of the adapting step to give one point on a rate-displacement curve [the $R(X)$ curve; Fig. $1 E]$. The process was then repeated with 14 other displacements to generate a complete $R(X)$ curve. The entire procedure involved about 1260 individual stimuli, usually presented at $5 / \mathrm{sec}$, so that one set took almost $5 \mathrm{~min}$. Thus, it was possible to do 15-30 solution changes while the preparation was stable.

Isolated hair cell preparation. Single, dissociated hair cells were obtaincd by a mild papain digestion of the sacculus followed by mechanical separation of cells, according to Assad et al. (1989). They adhered to the clean glass bottom of the recording chamber with sufficient strength that the cell bodies did not move when the bundles were stimulated. Patch pipettes were sealed to the basolateral surfaces, near the cuticular plate. The whole-cell membrane potential was controlled and transduction current measured by a patch clamp (Yale Mk V). Series resistance compensation limited the voltage error to less than $3 \mathrm{mV}$.

Hair bundles were mechanically stimulated by attaching a clean glass stimulus probe to the bulbs of kinocilia. The probe was moved in the horizontal plane by a 2-dimensional bimorph driver (Corey and Hudspeth, 1980), which was calibrated by observation of a viden image at $10,000 \times$ and by a photodiode in the camera image plane of the microscope (Zeiss IM-35).

The extracellular solution was a normal frog saline; the intracellular solution was designed to block the large $\mathrm{Ca}^{2+}$-activated $\mathrm{K}^{+}$conductance in these cells (Table 1). The chamber was continuously perfused with oxygenated saline at room temperature.

\section{Results}

\section{Time course of the adaptive shift}

When the ciliary bundles of hair cells in a patch of the macula were moved in a positive direction (towards their kinocilia), the current entering the apical surface of the cpithclium incrcascd, and then declined over the following $500 \mathrm{msec}$ (Fig. 2, $A, C, E$; lower traces). The decline of current was correlated with a shift along the displacement axis of the $I(X)$ curve for the preparation. Many different processes could lead to a decline in the net current, such as channel inactivation or the induction of another current. However, the shift of the $I(X)$ curve occurred with almost no change in its amplitude or vertical position, indicating that the general relationship between displacement and the 

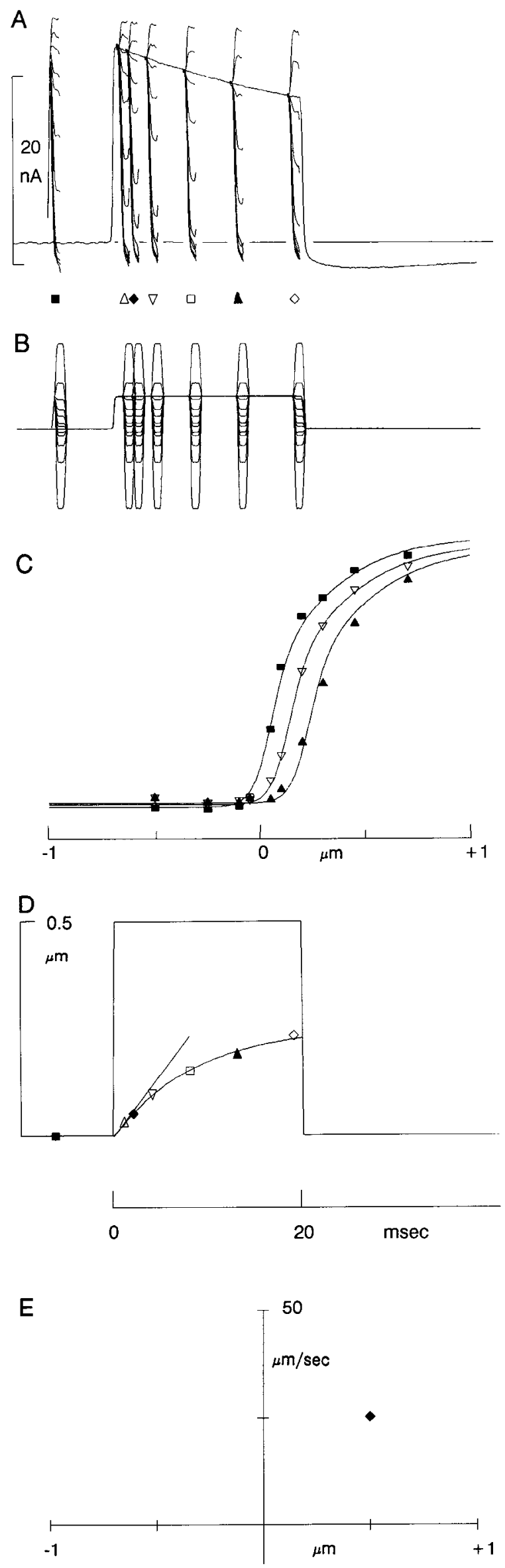

opening of channels was unchanged and that the decline must be due to a decrease in the tension stimulus applied to transduction channels. If the simple model of an elastic spring attached to the channel protein is correct (Corey and Hudspeth, $1983 \mathrm{~b}$ ), then the tension is the product of the spring constant and the elongation of the spring, and the slope of the $I(X)$ curve is related to the spring constant. Since the $I(X)$ curve shifted without substantial change in its slope (Fig. $1 C$; see also Assad et al., 1989, Fig. 3C), the attachment point of the spring must have moved, rather than the spring constant changing. This process constitutes an adaptation mechanism for bullfrog saccular hair cells, which allows cells to be sensitive to small changes in bundle position, while rejecting large static displacements (Eatock et al., 1987).

In Figure 2 (upper traces), the magnitude of the shift was plotted as a function of time, for comparison to the displacement of the bundles. If the shift results from the movement of an attachment point, then these plots also represent the movement of the attachment point, scaled by some geometry factor. For instance, if the tip-links model of transduction is correct, the geometry factor, $\gamma$, is about 0.1 (Howard and Hudspeth, 1987). A $+0.5 \mu \mathrm{m}$ displacement stimulus of the bundles then corresponds to a $50 \mathrm{~nm}$ elongation of the link. Following the displacement, the $I(X)$ curve shifted by about $0.25 \mu \mathrm{m}$ in the first $20 \mathrm{msec}$ following the stimulus (Fig. $2 A$ ). The attachment point would have moved by about $25 \mathrm{~nm}$ in a direction to relax the tension.

Negative stimuli that decreased the transepithelial current also caused a shift in the $I(X)$ curve. In Figure $2 B$, a $-0.5 \mu \mathrm{m}$ displacement turned off all the mechanically sensitive current (lower trace); during the first $20 \mathrm{msec}$ the $I(X)$ curve moved by about $0.1 \mu \mathrm{m}$ in the negative direction (upper trace). Although the current remained at zero throughout the $20 \mathrm{msec}$ adapting step, the pulse protocol to measure $I(X)$ curves revealed the shift, and it was further manifest as a rebound in the current at the termination of the step.

The time course of the $I(X)$ shift was fitted well by singleexponential functions, at least at short times (Fig. 2, $A, B$, upper traces). When the adapting steps were extended to $100 \mathrm{msec}$, the magnitudes of the shift were closer to the amplitudes of the adapting step; that is, the adaptation was more complete. However, a single-exponential function was not always an adequate fit: the shift at early times was faster than the best-fit single exponential, and the shift at later times was slower (Fig. 2C). With adapting steps of $2 \mathrm{sec}$ duration, the shift was still not

Figure 1. Protocol for measuring the initial rate as a function of displacement [the $R(X)$ curve]. $A$, Responses to the mechanical stimuli depicted in $B$. An adapting step of $0.5 \mu \mathrm{m}$ amplitude and $20 \mathrm{msec}$ duration was presented, with one superimposed test step of varying amplitude and delay. Test steps were varied over 12 amplitudes to generate an instantaneous $I(X)$ curve, and over 7 times relative to the adapting step to generate the time course. The repetition rate was $5 / \mathrm{sec}$ for most experiments, but always at least 10 times longer than the adapting step. $C$, At each time, the instantaneous $I(X)$ curves were plotted and compared with the resting $I(X)$ curve acquired before the adapting step. Shown are the resting curve (filled squares) and the curves at 8 and $13 \mathrm{msec}$ (open and filled triangles). The current scale is as in $A$. $D$, The resting $I(X)$ curve was shifted on the $X$-axis to match each instantaneous $I(X)$ curve, and the shift plotted against time. The rate was then determined from the initial slope of an exponential curve fitted to the time course. $E$, The rate was plotted as one point on an $R(X)$ curve. 
A
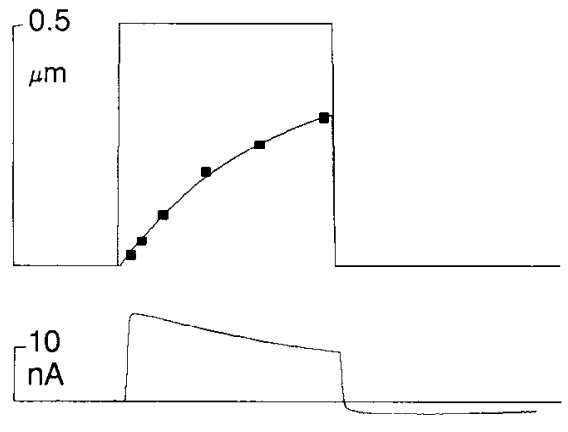

B
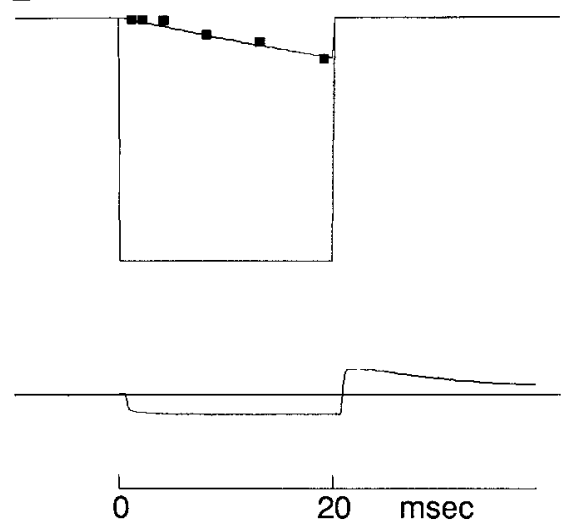

C

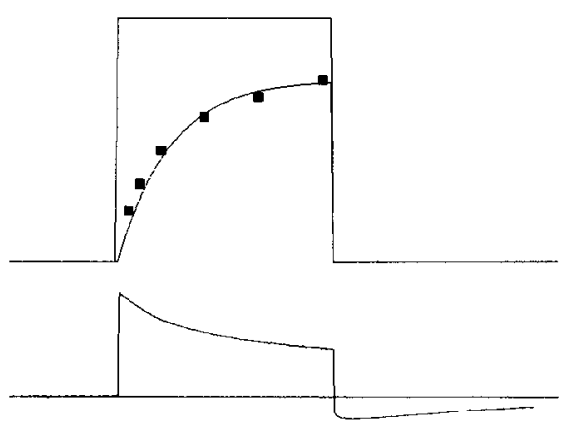

D
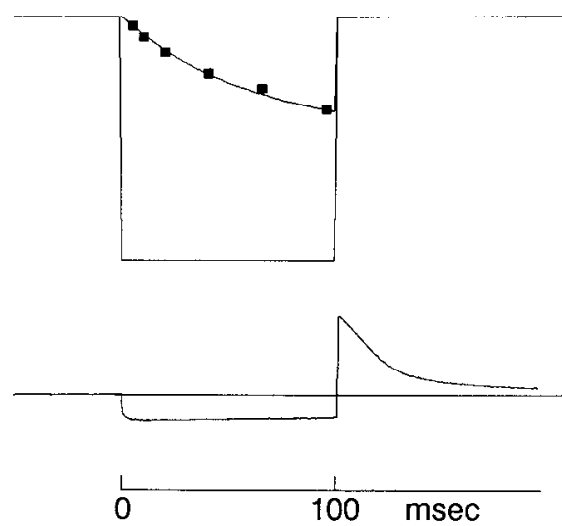

$\mathrm{E}$

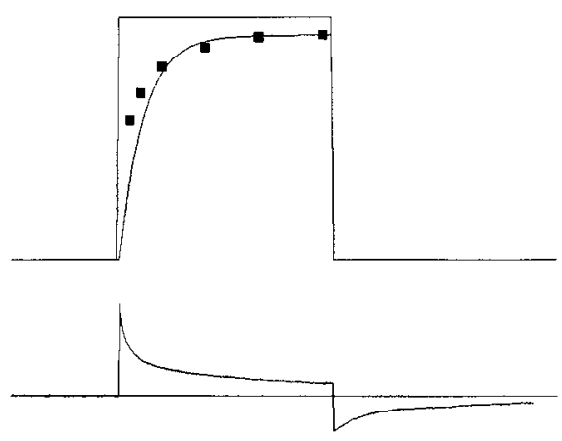

$\mathrm{F}$
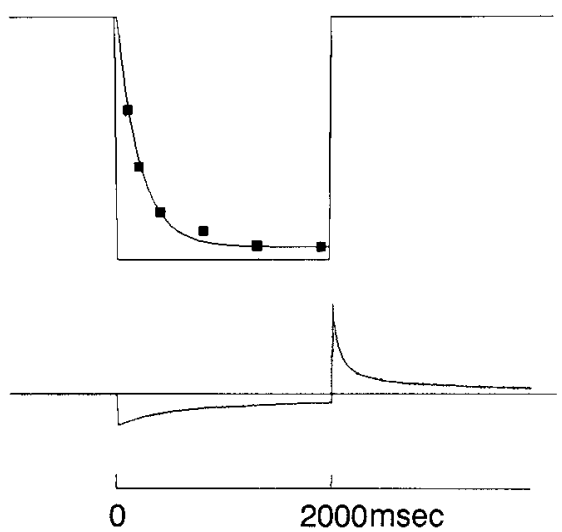

Figure 2. Time course of the adaptive shift for short and long adapting steps. For each panel, the lower trace is the time course of current across the epithelium, with current entering transduction channels shown as upwards. The baseline represents the current flowing through channels open at rest; downward deflections indicate those channels closing. The upper part of each panel is the time course of the $I(X)$ curve shift, and for comparison, the size and duration of the adaptation step. Complete adaptation, in which channels return to their open probability at rest, occurs if the shift equals the displacement. Amplitude scales are the same throughout; the time course is shown for each pair of positive and negative steps. Apical solution was $1 \mathrm{mM} \mathrm{Ca}^{2+}$ endolymph.

complete, and a single-exponential function was not an adequate description of the time course (Figs. 2, E, F).

One possible explanation for the inadequacy of the singleexponential fit is that the shift mechanism may consist of several processes: a fast tension adjustment system followed by a slower rearrangement of cytoskeletal structures. Another is that the rate of the shift seems to be regulated in part by calcium entering transduction channels and acting at an intracellular site (Assad et al., 1989). Thus, calcium entry, and the rate, would be greatest when channels were first opened but less as they closed during the adapting step.

\section{Initial rate of the adaptive shift}

Regardless of the reason, we felt the analysis would be simplified if we restricted our attention to the rate of the shift in the first few milliseconds following the onset of the adapting step. The rate would then reflect the fast process(es) of the system for tension regulation, and it would reflect the rate of movement before much had changed: before the attachment point had moved into saturating regions of its range and before the tension in the spring had changed substantially.

For this analysis, a single-exponential curve was fitted to the points describing the shift as a function of time (Fig. 1D), and the rate was taken as the initial slope of that curve (calculated as the amplitude of the exponential divided by the time constant). While it is notoriously difficult to extract accurate parameters from exponential fits to limited data, this method is less sensitive to fitting errors: various fits tend to produce the same initial slope. Indeed, essentially the same rates could be obtained by a linear least-squares fit to the first 3 points.

Figure $3 A$ shows the relation between initial rates of shift and the size of the adapting step. This $R(X)$ curve describes the shift of the $I(X)$ curve as a function of the displacement of the hair bundles; if the tip-links model is correct, it also describes the rate of movement of the attachment point (at rates and displacements about 10-fold less). For comparison, the $I(X)$ curve for this preparation is shown on the same displacement scale (Fig. 3B).

Several features of the tension regulation mechanism are apparent from the $R(X)$ relation. First, the positive rate, which reflects relaxation following a positive stimulus, was generally larger than the negative rate, which reflects retensioning following a negative stimulus. This confirms the asymmetry of rates inferred from the $I(X)$ shift following a tone burst stimulus (Eatock et al., 1987). The physiological consequence of the asymmetry is that an oscillatory stimulus would tend to produce 

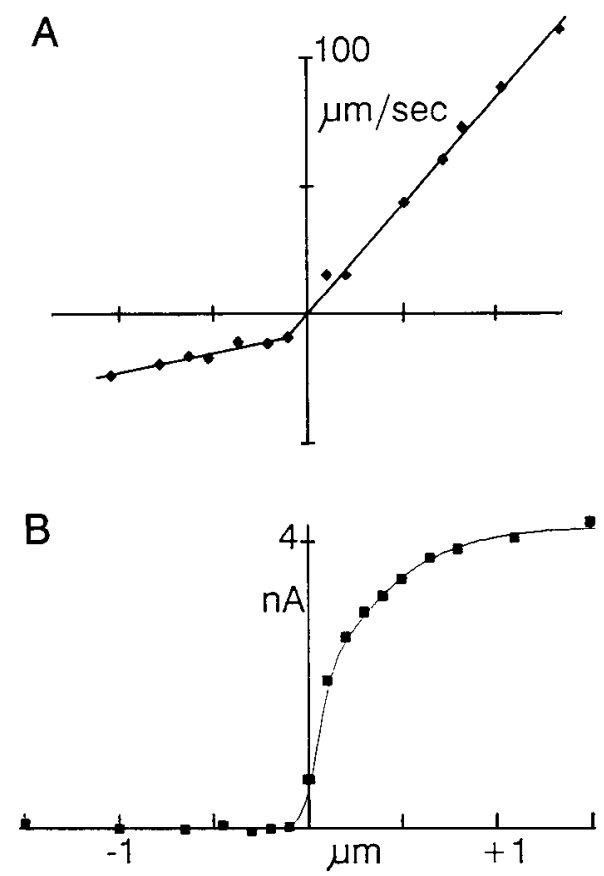

Figure 3. $A$, The $R(X)$ curve obtained by plotting initial rates of the shift as a function of the size of the adapting step. $B$, The $I(X)$ curve for the same preparation, with the minimum current taken as zero. The current scale is rather arbitrary, as it was not known exactly how many cells remained attached to the otolithic membrane and contributed to the current. The displacement scale for $A$ is as for $B$. Apical solution was $1 \mathrm{mM} \mathrm{Ca}^{2+}$ endolymph.

a negative (inhibitory) shift of the $I(X)$ curve, because the relaxation during the positive phase of the stimulus would occur more rapidly than the retensioning during the negative phase.

Second, the positive rate was roughly linear with displacement. Since the tension in the elastic gating spring is expected to be roughly linear with displacement, this suggests that the positive (relaxation or slipping) rate is primarily determined by tension in the gating spring. One problem with this interpretation is that the rate is also known to be sensitive to calcium entry (see below, and Assad et al., 1989) and that larger displacements lead to increased channel opening and more calcium entry. Nevertheless, the rate continued to increase with displacement past the point on the $I(X)$ curve where all channels were open. Thus, a major (though not the sole) determinant of positive rate is tension on the mechanism.

Third, the negative rate was less sensitive to displacement. Not only was the rate less at most displacements, but the rate did not change much between small and large negative displacements. Indeed, the tip-links model is consistent with a negative rate completely independent of displacement: Beyond a certain negative displacement, the links might be slack, and the rate would then be the intrinsic, or zero-tension, rate of the mechanism that moves the attachment point. It is perhaps surprising, then, that the negative rate was rarely completely independent of displacement in this preparation but usually had some positive slope.

\section{Initial rate in an isolated-cell preparation}

It is possible that the displacement dependence of the negative rates is a population effect-an artifact of the microphonic preparation. If some cells were poorly coupled to the otolithic mem- brane, for instance, their $I(X)$ curves would be very broad and a large negative displacement might be required to make their tip-links slack. Thus, their adaptation might be displacementdependent over a wide range, and they would contribute a displacement-dependent component to the overall response of the preparation.

To check for population artifacts, the negative rates were measured in single cells that were voltage-clamped with a patchclamp amplifier and stimulated under direct visual observation. Because the preparation was not sufficiently stable over the minutes required for the complete $R(X)$ protocol, the negative rates were calculated from the rebound in current at the termination of a $25 \mathrm{msec}$ negative displacement step. The rebound current was compared with the resting $I(X)$ curve, measured with a triangle-wave stimulus immediately beforehand, in order to determine how far the $I(X)$ curve must have shifted during the negative step. Rates were taken as the average rate over $25 \mathrm{msec}$, which is not greatly different from the initial rate since the time constants for negative steps were long.

In 15 out of 17 cells that were judged to be firmly attached to the chamber bottom, the negative rate of adaptation was independent of displacement for larger negative displacements (Fig. 4). The steepness of the $I(X)$ curve varied among cells in this preparation; for both broad and narrow curves it appeared that the adaptation rate became independent of displacement at or just beyond the negative saturation of the $I(X)$ curve. We expect that these results from single cells are more representative of the adaptation mechanism and that the negative rates are independent of displacement in a range where the tip links may be expected to be slack.

\section{Calcium dependence of the rates}

The asymmetry of rates suggests that the relaxation and retensioning are 2 qualitatively different processes-or at least quantitatively different aspects of the same process. There is evidence that the relaxation is a passive, force-dependent slipping process and that the retensioning is an active, force-generating motile mechanism (Howard and Hudspeth, 1987; Assad et al., 1989), perhaps involving the same molecular motor.

Can these processes be further separated? In the microphonic preparation, calcium in the solution bathing hair bundles accelerates the rate of adaptation (Eatock et al., 1987), apparently by entering through transduction channels to act at an intracellular site (Assad et al., 1989). Figure 5 shows the change in the $R(X)$ relations with increased calcium in the apical solution (see Table 1). At $25 \mu \mathrm{M}$ extracellular calcium (Fig. 5, $A, B$ ), the positive and negative rates of shift were both fairly linear with displacement, and low. The $I(X)$ curve was shifted somewhat to the left, implying an increased resting tension in the gating springs. At higher calcium concentrations (100 $\mu \mathrm{M}$ in Fig. 5, $C$, $D$ and $1 \mathrm{~mm}$ in Fig. $5, E, F$ ), the positive rates increased at all displacements, while the negative rate increased, but less so. Concurrently, the resting $I(X)$ curves moved to the right, in the direction of lower resting tension. At $10 \mathrm{~mm}$ calcium, the positive rates were higher still, at least for displacements up to a few tenths of a micrometer, while the negative rates remained less dependent on displacement. The $I(X)$ curve at $10 \mathrm{~mm}$ indicated still less resting tension. (In $10 \mathrm{~mm}$ calcium, the positive rates at larger displacements can be seen to depart from a linear relationship, becoming less than for equivalent displacements in lower calcium. This was a consistent observation in all experiments, but the mechanism for it is not clear.) 
A

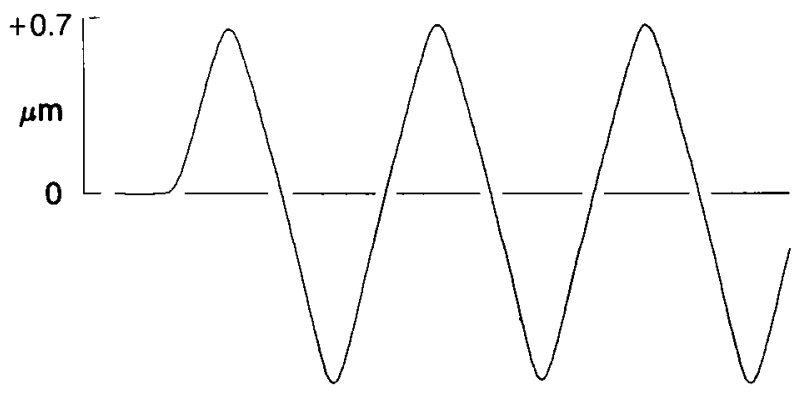

B

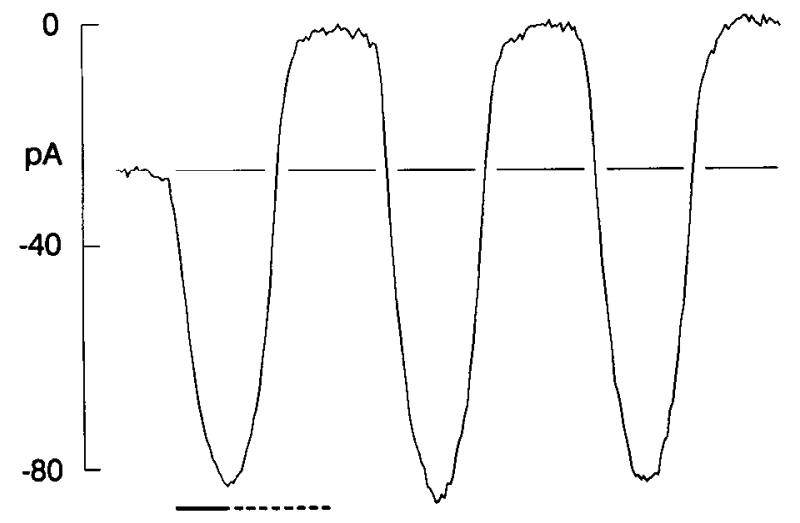

C

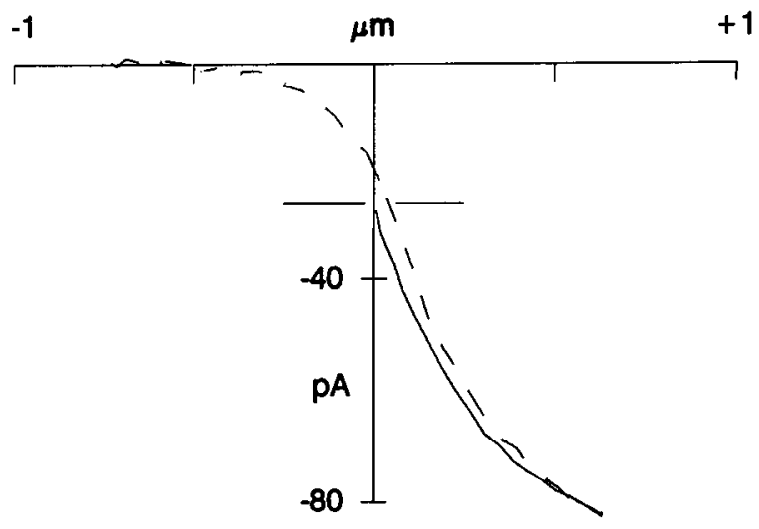

D

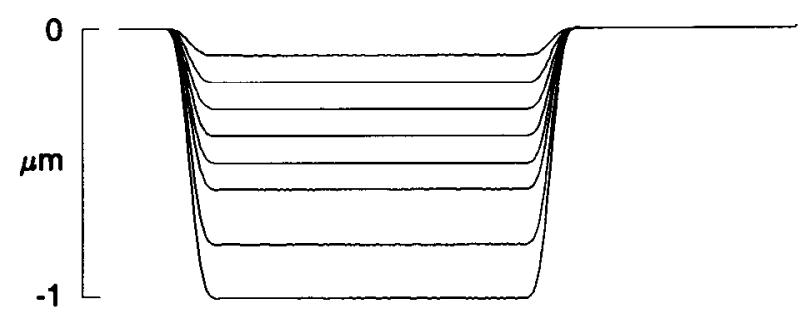

E

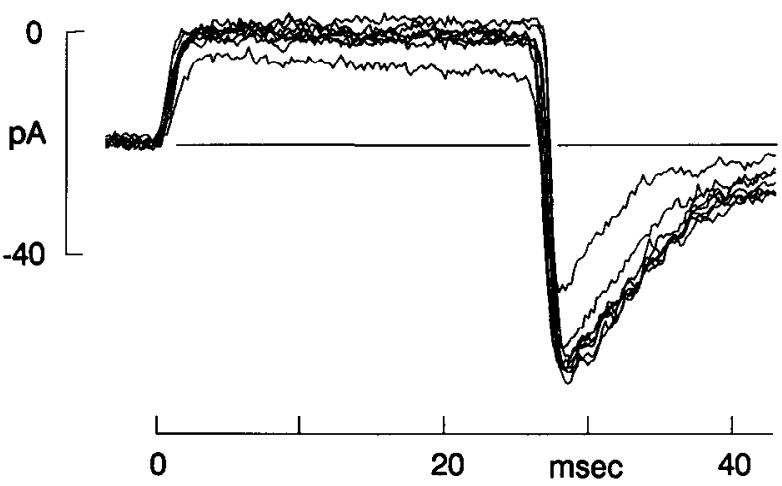

F 1

Figure 4. Negative rates measured in single cells, by comparing the rebound following negative steps to the $I(X)$ curve. $A$, A triangle-wave stimulus delivered to the bundle of a single, isolated hair cell elicted a change in the transduction current, $B$, measured by whole-cell patch-clamp recording. Inward current is shown as downward in this figure; the saturation with negative displacements is assumed to be zero transduction current. The time scale is as in $E$. $C$, An $I(X)$ curve was generated by plotting the current against the displacement for $3 / 4$ of one cycle. The initial increase in inward current is shown as solid, while the declining half-cycle is dashed, to correspond to the line indicating the plotted region of the response in $B . D$, A series of negative adapting steps was presented to the same cell; $E$, each (except for the smallest step) was sufficient to turn off the transduction current. At the termination of each step there was a rebound in the inward current, indicating that the $I(X)$ curve had shifted to the left during the negative displacement. $F$, The amount of rebound current was compared with the $I(X)$ curve to determine how much the $I(X)$ curve had shifted; this was divided by the step duration to get an average rate. Rates were plotted against the size of the adapting step.

The dependence of the rates on calcium for 5 such experiments is summarized in Figure 6. For each concentration, the slope of the $R(X)$ relation was measured for positive and negative displacements, and plotted against concentration. This further illustrates the differential dependence of the rates on calcium: the negative slopes were largely independent of calcium, while the positive rates more than doubled as calcium was raised. (Although lines fitted to the negative rates did not generally intercept the origin, the intercepts were similarly insensitive to calcium.)

It should be emphasized that the relation between extracellular calcium and the slopes is somewhat removed from the specific calcium dependence of the intracellular mechanism: surface concentration of calcium near the mouth of the channeland thus calcium influx-is unlikely to be linearly related to calcium in bulk solution, and intracellular calcium is a com- 

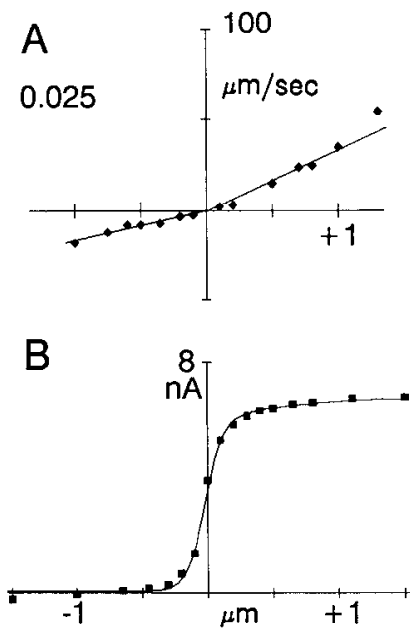
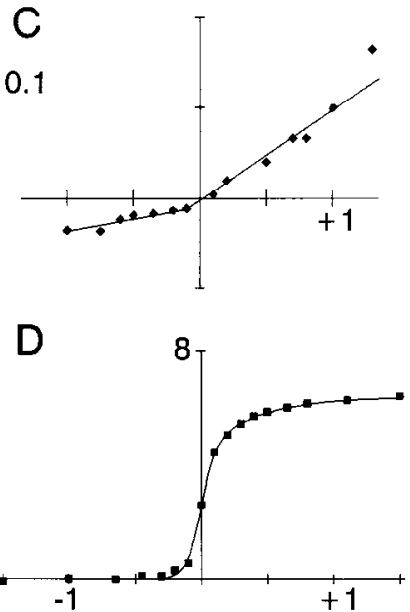
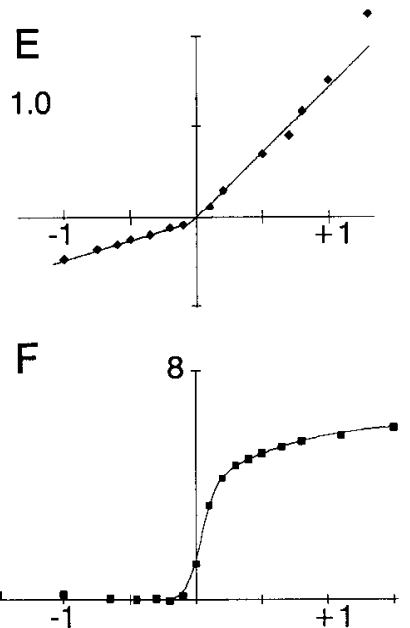

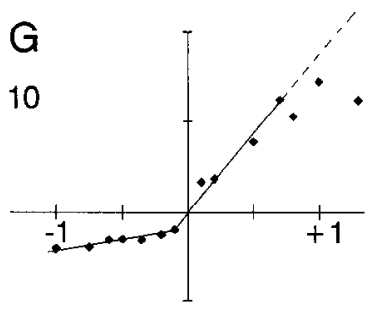

$\mathrm{H}$

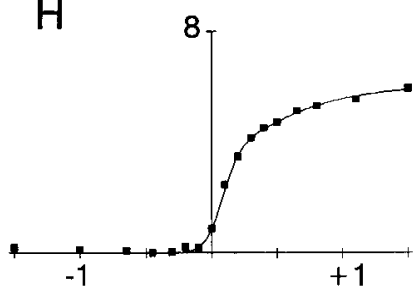

Figure 5. $R(X)$ and $I(X)$ curves at the 4 calcium concentrations indicated. As calcium was increased, the rates for positive shifts increased but the negative rates were less affected. The $I(X)$ curves were shifted to the right in higher calcium, indicating reduced resting tension. Solutions are given in Table 1.

plicated function of resting influx and the change in influx when channels are stimulated. This is more an operational description of an intact system. The qualitative relation seems clear, however: The positive rates are changed by calcium, while the negative rates are mostly unchanged.

Moreover, it suggests a mechanism for the calcium control of the resting tension, as inferred from the resting position of the $I(X)$ relation. The equilibrium tension may result from a balance between relaxation and tensioning processes (e.g., Howard and Hudspeth, 1987; Assad et al., 1989), so that changing

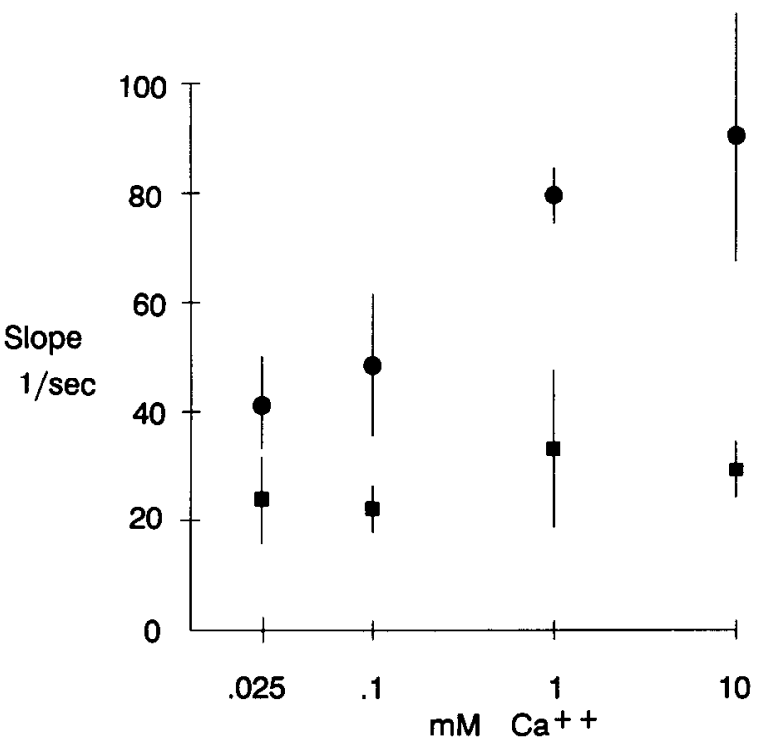

Figure 6. Slopes of the positive (circles) and negative (squares) parts of the $R(X)$ curves measured in 4 calcium concentrations. Lincs werc fitted to the points by eye, and the slopes measured in units of $\mathrm{sec}^{-1}$. For negative rates the fitted line often did not pass through the origin, but the $Y$-intercepts also did not change much with calcium. For positive rates in $10 \mathrm{~mm}$ calcium, the line was fitted to the rates up to about 0.4 $\mu \mathrm{m}$, so the saturation in rate often observed in high calcium is not represented here. Points represent the average and SDs from 5 experiments. either rate would change the equilibrium. Calcium's potentiation of the relaxation rate would be expected to shift the equilibrium towards reduced tension, and to shift the $I(X)$ curve to the right, as was observed.

\section{Ionic specificity of calcium action}

If calcium is acting at an intracellular site, is it a calcium-specific binding site or could it be a nonspecific charge effect? Figure 7 compares the effects of 4 divalent cations on the adaptation rates. In each case, the rates were measured in an apical solution with $0.1 \mathrm{~mm}$ calcium, and then $1 \mathrm{~mm}$ of the test divalent was added to the control solution and the rates remeasured. In Figure $7, A, B$, the expected calcium effect occurred: the addition of 1 . mM calcium increased the positive rates and shifted the $I(X)$ curve to the right. (In the experiment of this figure the peak current was also increased slightly; usually added calcium lowered the current slightly, as in Fig. 5, $D, F$.) When barium was added, however, the rates were if anything reduced (Fig. 7C). Magnesium reduced the positive rates more (Fig. $7 E$ ). Both barium and magnesium reduced the peak current, but for both the proportion of current active at rest was increased, that is, the $I(X)$ curves were shifted to the left (Fig. 7, D, F). Strontium exerted the most pronounced effect: the positive rates were substantially reduced, and the $I(X)$ curve was shifted to the left so that most of the channels were open at rest (Fig. 7, $G, H$ ).

Thus, these 3 divalent cations did not substitute for calcium in potentiating the positive adaptation rate; instead, they appeared to compete with the $0.1 \mathrm{~mm}$ calcium in the control solution to inhibit the relaxation. It could be that they compete with calcium in the channel to reduce calcium entry. However, since all 3 can pass through the transduction channel (Corey and Hudspeth, 1979; Ohmori, 1985) and since the calciumdependent site is intracellular (Assad et al., 1989), the intracellular site must have a specificity for calcium. As calmodulin has been found to be preferentially localized within the tips of the stereocilia (Shepherd et al., 1989), it could be the regulatory site. Yet there is only indirect physiological evidence for an involvement of calmodulin in the adaptation (Corey et al., 1987), and there are other calcium-binding proteins within stereocilia (Shepherd et al., 1989). 

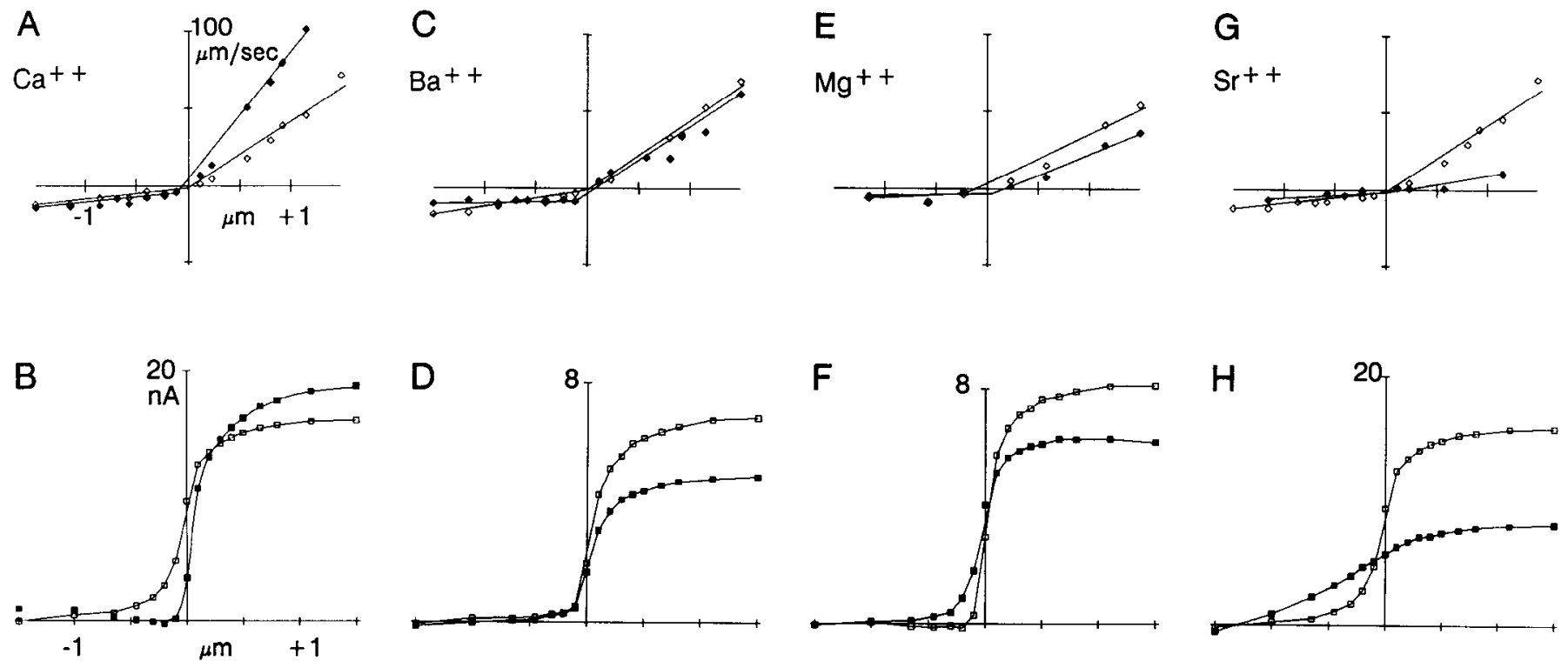

Figure 7. Ionic specificity of the calcium effect on adaptation. For each experiment, $R(X)$ and $I(X)$ curves were measured first in a 0.1 mM Ca ${ }^{2+}$ endolymph control solution (open squares), then the solution was changed to a solution that was the control plus $1 \mathrm{~mm}$ of the test divalent cation (filled squares). Effects were fully reversible.

\section{Aminoglycoside block of the adaptative shift}

Because of the ototoxicity of the aminoglycoside antibiotics, the effects of streptomycin and its analogs have been studied in a number of hair-cell preparations. Among other actions, the aminoglycosides produce a strongly voltage-dependent block of the transduction channel, implying a blocking site within the pore (Hudspeth and Kroese, 1983; Ohmori, 1985). In addition, gentamycin blocks the gating compliance of the bundle, apparently preventing the channel from either opening or closing (Howard and Hudspeth, 1988).

We found that low doses of dihydrostreptomycin had a pronounced effect on the relaxation rate of the shift (Fig. 8). Addition of $10 \mu \mathrm{M}$ of the drug to a solution containing $1 \mathrm{mM}$ calcium reduced the rates for positive displacements in this experiment 2-fold: the slope was decreased from 50 to $26 \mathrm{sec}^{-1}$, a change comparable to that produced by reducing calcium by more than 10 -fold. The rates for negative displacements were unchanged. The differential effect on the rates was also manifest as a shift of the $I(X)$ curve: dihydrostreptomycin increased the proportion of channels open at rest from 21 to $33 \%$ in this experiment.

The peak current in the $I(X)$ relation was reduced only slightly, by about $20 \%$ (Fig. $8 D$ ). If channel block were the main action of the aminoglycosides, it would be necessary to block the channels by $90 \%$ to effect a 10 -fold reduction in calcium entry. As dihydrostreptomycin is highly charged ( +3 at physiological $\mathrm{pH}$ ), it seems unlikely that it could cross the cell membrane to act intracellularly.

It might be thought that dihydrostreptomycin reduced calcium entry by screening negative charge that would otherwise accumulate calcium near the mouth of the channel. For instance, the surface potential can be calculated from the Grahame equation if an estimate is made of surface charge density (Hille, 1984). Calcium accumulation can then be determined from the surface potential and the Boltzmann relation. A decrease in the surface potential by the addition of a charged ion would decrease the calcium accumulation. It can be shown, however, that for
$10 \mu \mathrm{M}$ dihydrostreptomycin to cause a 10 -fold reduction in calcium at the mouth of the channel, the equivalent surface charge density would have to be about $3.2 \mathrm{chg} / \mathrm{nm}^{2}$. Studies on other channels suggest instead a substantially lower density of $0.05-$ $0.1 \mathrm{chg} / \mathrm{nm}^{2}$ (e.g., Dani and Eisenman, 1987). Thus, the streptomycin effect may involve a more specific binding interaction. It is not channel block and is unlikely to be a simple chargescreening effect within the mouth of the channel.
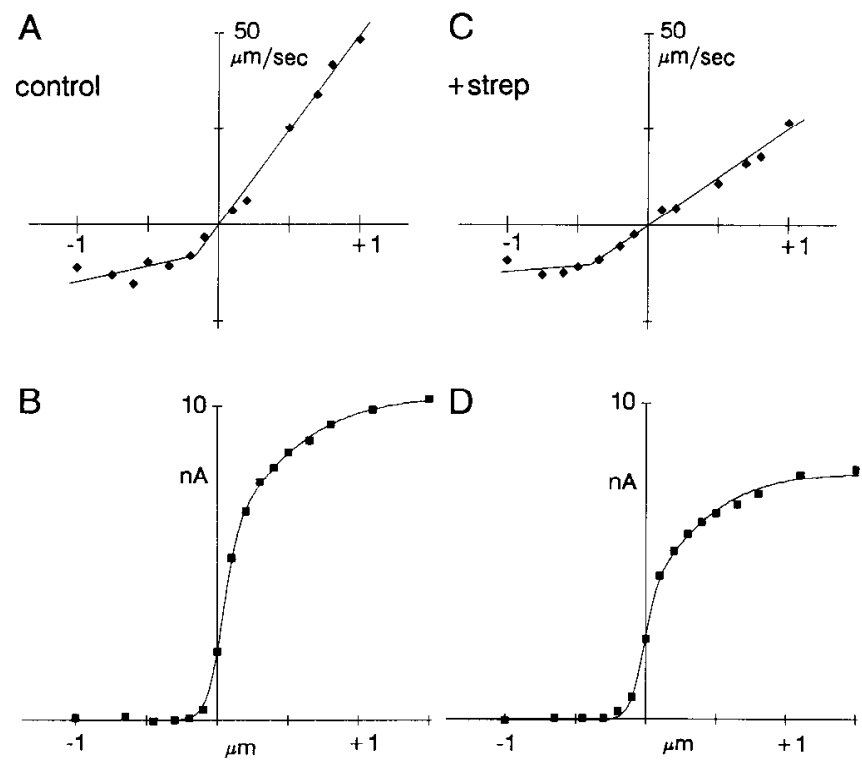

Figure 8. Effect of dihydrostreptomycin on the rates. $R(X)$ and $I(X)$ curves were measured in $1 \mathrm{~mm}$ calcium $(A, B)$ and then with $10 \mu \mathrm{M}$ dihydrostreptomycin added $(C, D)$. The drug reduced the positive rates and shifted the $I(X)$ curve to the left. Higher concentrations of dihydrostreptomycin nearly abolished the adaptation but reduced the total current more. 


\section{Discussion}

The adaptation mechanism for transduction in bullfrog saccular hair cells can be described operationally as a shift of the $I(X)$ curve along the $X$-axis in response to displacements of the bundle (Corey and Hudspeth, 1983a; Eatock et al., 1987). If certain assumptions are made about the transduction mechanism, characterization of the proccss leads to some fairly specific inferences about the adaptation mechanism.

Mechanical transduction by hair cells was proposed to result from direct application of tension to a channel protein by some elastic element (Corey and Hudspeth, 1983b). There is now considerable evidence for this model, which includes a direct measurement of the elasticity of the element, now termed the "gating spring" (Howard and Hudspeth, 1987, 1988). In this model, the steepness of the $I(X)$ curve is dependent on the stiffness of the gating spring, $\kappa_{G}$, and the position of the curve on the displacement axis depends on the resting tension in the spring (Corey and Hudspeth, 1983b; see also Howard et al., 1988). We have observed that the adaptive shift of the $I(X)$ curve occurs without a substantial change in its slope (Fig. $1 C$; Eatock et al., 1987; Assad et al., 1989), so if this model is correct, the shift corresponds to a change in the resting tension that is mediated by a change in the attachment point, and not by a change in the stiffness.

[We can imagine one other way that the shift might occur: a change in the relative energies of the open and closed states of the channel might arise from an enzymatic modification of the channel protein and would appear as a shift on the $X$-axis. Such a mechanism would have to involve multiple sites for modification, as the shift appcars to be continuous over a broad displacement range. Moreover, it would not explain the observed mechanical relaxations of the bundle that are correlated with adaptation (Howard and Hudspeth, 1987)].

A more specific transduction model has followed from the additional localization of the ion channels to the tips of the stereocilia (Hudspeth, 1982) and from the observation of fine filaments ("lip links") extending from the tip of each stereocilium to the side of its taller, adjacent neighbor (Pickles et al., 1984). It supposes that the morphological tip links are the physiological gating springs - that they are attached directly to the channel proteins in the tips. While there is as yet little direct evidence for this model, it has an extraordinarily elegant simplicity and is consistent with all current physiological data.

If the tip-links model is correct, then the movement of the attachment point may correspond to a movement of one end of the tip link along the side of the stereocilium. This was put forth as a speculative model by Howard and Hudspeth (1987) and is made more likely by the observations here. Alternative models involving gross rearrangement of the stereocilia or the cuticular plate are not consistent with direct visual or video observation of adapting cells (Eatock et al., 1987; Corey et al., 1989).

The geometry of the bundle then permits a calculation of the actual rate of movement along the stereocilium: positive displacements of the tip of the bundle would stretch the tip links by a factor $(\gamma)$ less, and the real rates would be less by the same factor. $\gamma$ has been estimated as about 0.14 by Howard and Hudspeth (1987) and is nearly that in our calculations (Corey et al., 1989). Thus, the initial rate of movement along the stereocilium in $1 \mathrm{~mm}$ calcium was $1-2 \mu \mathrm{m} / \mathrm{sec}$ in the negative direction, which corresponds to tensioning or to the high end climb- ing up the stereocilium. The positive rate, which corresponds to relaxation or to slipping down, ranged up to $13 \mu \mathrm{m} / \mathrm{sec}$ along the stereocilium in these experiments but would presumably have been larger if larger displacements had been presented.

Are these reasonable rates for biological processes? The tensioning rate is very close to that of myosin moving on actin in an in vitro system (2-6 $\mu \mathrm{m} / \mathrm{sec}$; Sheetz et al., 1984) and to kinesin moving on microtubules $(0.5-1.6 \mu \mathrm{m} / \mathrm{sec}$; Vale et al., 1985).

If the tip links are anchored to transmembrane structures, these structures would be dragged through the lipid during the movement. The limiting velocity for protein movement in lipid is given by $V=F D / k T$, where $F$ is the force parallel to the lipid, $D$ is the diffusion constant, and $k T$ is as usual. A positive $1 \mu \mathrm{m}$ displacement is expected to produce a tension of roughly $80 \mathrm{pN}$ in each tip link (Howard and Hudspeth, 1987), and rhodopsin's diffusion constant in rod outer segments is $4 \times 10^{-9} \mathrm{~cm}^{2} / \mathrm{sec}$. If this were the limit to velocity, the transmembrane element could move at about $8000 \mu \mathrm{m} / \mathrm{sec}$, more than 500 -fold faster than the maximum observed rate. Lipid drag is apparently not rate-limiting.

What does determine the rate of movement of the attachment point? It seems quite plausible that the negative rate is that of a molecular motor actively climbing on the actin core of the stereocilium (Howard and Hudspeth, 1987); actin in the stereocilium is oriented such that myosin would move up it towards the tip (Flock et al., 1981). A suggestion that the motor is the myosin-like $110 \mathrm{kDa}$ protein of intestinal brush border (Howard and Hudspeth, 1987) seems not to be true: the $110 \mathrm{kDa}$ protein is apparently not in frog hair-cell stereocilia, based on gel electrophoresis (Shepherd et al., 1989) and immunohistochemistry (unpublished observations). The motor may nevertheless be a member of the myosin I family. Attachment of a channel to a myosin-like motor would also provide a way to position channels at the tips of the stereocilia: myosin moving on the side of the actin core would naturally carry them up.

A confusing aspect of the negative rate for this model is that it continued to depend on displacement in the microphonic preparation, even for large negative displacements that might be expected to make the tip links slack. The mechanism should not be able to sense bundle displacement if the links are truly slack. The absence of such dependence in single cells, when bundles were directly stimulated, argues that this is a population effect, perhaps due to poor coupling to some bundles in the microphonic preparation. In electron micrographs of bullfrog saccular hair cells the tip links appear to be about $100 \mathrm{~nm}$ long (J. A. Assad and D. P. Corey, unpublished observations), so that negative displacements of $1.0 \mu \mathrm{m}$ (with a $\gamma$ of 0.14 ) are likely to make them slack. Howard and Hudspeth (1988) did measure in some cases a decrease in bundle compliance with negative displacements of a few hundred nanometers, which could correspond to a slackening. Thus, it seems likely that the larger negative displacements we presented exceeded the shortening limit of the tip link, and we might expect zero tension in this range. The negative rate in this range would correspond to the intrinsic rate of the molecular motor.

The positive rates are difficult to interpret from these data because positive displacements increase tension on the spring but also open channels to let in more calcium. In the region of the $I(X)$ curve where the current is saturated, however, the rates are clearly linear with displacement, and thus linear with tension if the spring follows Hooke's law. It seems fairly reasonable that slipping might be linear with tension: if the motor was bound 
to its substrate part of the time (as it must be during an active stroke), but unbound part of the time, it would slip a certain distance while it was unbound and that distance might be linear with tension.

In any case, the linear rates at large displacements are evidence that the displacement sensitivity of the adaptation motor does not follow from the displacement sensitivity of the channel (Eatock et al., 1987) and that the motor (as well as the channel) senses tension in the spring. In this protocol, indeed, we are using the channel as a tension sensor to study the tension dependence of the motor.

Calcium potentiated the positive (slipping) rate while affecting the negative rate much less. If the notion is correct that slipping depends on the time the motor is not bound to the substrate, then calcium could be imagined to increase the unbound time. The site for calcium's action on the rates is specific for calcium, as other divalents either did not substitute or inhibited the action of calcium. This site appears to be inside the tips of the stereocilia (Assad et al., 1989).

These experiments suggest that 2 processes act to regulate the resting tension on transduction channels. In the primary process, the equilibrium is set at the point where the (tension-dependent) relaxation rate matches the (tension-independent) tensioning rate; that is, the motor tightens until it slips faster than it tightens. Reduced extracellular calcium cause a lower relaxation rate at any given tension, so in low calcium the motor tightens more before the equilibrium is reached. The result would be more channels open at rest in reduced calcium, as was observed.

A secondary effect results from calcium entry following channel opening. In this process, the motor increases tension on the channels, until they began to open and let calcium into the stereocilia. Then the calcium accelerates the relaxation and allows channels to close. Because the calcium dependence is generally less pronounced than the tension dependence, this effect of calcium entry adds additional feedback to a system that is primarily tension-dependent.

\section{References}

Assad, J. A., N. Hacohen, and D. P. Corey (1989) Voltage dependence of adaptation and active bundle movement in bullfrog saccular hair cells. Proc. Natl. Acad. Sci. USA 86: 2918-2922.

Corey, D. P., and A. J. Hudspeth (1979) Ionic basis of the receptor potential in a vertebrate hair cell. Nature $281: 675-677$.

Corey, D. P., and A. J. Hudspeth (1980) Mechanical stimulation and micromanipulation with piezoelectric bimorph elements. J. Neurosci. Meth. 3: 183-202.
Corey, D. P., and A. J. Hudspeth (1983a) Analysis of the microphonic potential of the bullfrog's sacculus. J. Neurosci. 3: 942-961.

Corey, D. P., and A. J. Hudspeth (1983b) Kinetics of the receptor current in bullfrog saccular hair cells. J. Neurosci. 3: 962-976.

Corey, D. P., W. J. Smith, B. A. Barres, and W. J. Koroshetz (1987) Calmodulin inhibitors block adaptation in vestibular hair cells. Soc. Neurosci. Abstr. 13: 149.1.

Corey, D. P., N. Hacohen, P. L. Huang, and J. A. Assad (1989) Hair cell stereocilia bend at their bases and touch at their tips. Soc. Neurosci. Abstr. 15: 89.6.

Dani, J. A., and G. Eisenman (1987) Monovalent and divalent cation permeation in acetylcholine receptor channels. J. Gen. Physiol. 89: 959-983.

Eatock, R. A., D. P. Corey, and A. J. Hudspeth (1987) Adaptation of mechanoelectrical transduction in hair cells of the bullfrog's sacculus. J. Neurosci. 7: 2821-2836.

Flock, A., H. C. Cheung, B. Flock, and G. Utter (1981) Three sets of actin filaments in sensory cells of the inner ear. Identification and functional orientation determined by gel electrophoresis, immunofluorescence and electron microscopy. J. Neurocytol. 10: 133-147.

Hille, B. (1984) Ionic Channels of Excitable Membranes, p. 321, Sinauer, Sunderland, MA.

Howard, J., and A. J. Hudspeth (1987) Mechanical relaxation of the hair cell bundle mediates adaptation in mechanoelectrical transduction by the bullfrog's saccular hair cell. Proc. Natl. Acad. Sci. USA 84: 3064-3068.

Howard, J., and A. J. Hudspeth (1988) Compliance of the hair cell bundle associated with gating of mechanoelectrical transduction channels in the bullfrog's saccular hair cell. Neuron $1:$ 189-199.

Howard, J., W. M. Roberts, and A. J. Hudspeth (1988) Mechanoelectrical transduction by hair cells. Annu. Rev. Biophys. Biophys. Chem. 17: 99-124.

Hudspeth, A. J. (1982) Extracellular current flow and the site of transduction by vertebrate hair cells. J. Neurosci. 2: 1-10.

Hudspeth, A. J., and D. P. Corey (1977) Sensitivity, polarity, and conductance change in the response of vertebrate hair cells to controlled mechanical stimuli. Proc. Natl. Acad. Sci. USA 74: 24072411.

Hudspeth, A. J., and A. B. A. Kroese (1983) Voltage-dependent interaction of dihydrostreptomycin with the transduction channels in bullfrog sensory hair cells. J. Physiol. (Lond.) 345: 66P.

Ohmori, H. (1985) Mechano-electrical transduction currents in isolated vestibular hair cells of the thick. J. Physiol. (Lond.) 359: 189217.

Pickles, J. O., S. D. Comis, and M. P. Osborne (1984) Cross-links between stereocilia in the guinea pig organ of Corti, and their possible relation to sensory transduction. Hear Res. 15: 103-112.

Sheetz, M. P., R. Chasan, and J. A. Spudich (1984) ATP-dependent movement of myosin in vitro: Characterization of a quantitative assay. J. Cell Biol. 99: 1867-1871.

Shepherd, G. M. G., B. A. Barres, and D. P. Corey (1989) "Bundle blot" purification and initial protein characterization of hair cell stereocilia. Proc. Natl. Acad. Sci. USA 86: 4973-4977.

Vale, R. D., B. J. Schnapp, T. S. Reese, and M. P. Sheetz (1985) Organelle, bead, and microtubule translocations promoted by soluble factors from the squid giant axon. Cell 40: 559-569. 